\title{
REVIEW
}

\section{Clinical review: Update on hemodynamic monitoring - a consensus of 16}

\author{
Jean-Louis Vincent ${ }^{1 *}$, Andrew Rhodes², Azriel Perel ${ }^{3}$, Greg S Martin'4 , Giorgio Della Rocca ${ }^{5}$, Benoit Vallet ${ }^{6}$, \\ Michael R Pinsky?, Christoph K Hofer ${ }^{8}$, Jean-Louis Teboul ${ }^{9}$, Willem-Pieter de Boode ${ }^{10}$, Sabino Scolletta ${ }^{11}$, \\ Antoine Vieillard-Baron'2, Daniel De Backer', Keith R Walley ${ }^{13}$, Marco Maggiorini14 and Mervyn Singer ${ }^{15}$
}

\begin{abstract}
Hemodynamic monitoring plays a fundamental role in the management of acutely ill patients. With increased concerns about the use of invasive techniques, notably the pulmonary artery catheter, to measure cardiac output, recent years have seen an influx of new, less-invasive means of measuring hemodynamic variables, leaving the clinician somewhat bewildered as to which technique, if any, is best and which he/she should use. In this consensus paper, we try to provide some clarification, offering an objective review of the available monitoring systems, including their specific advantages and limitations, and highlighting some key principles underlying hemodynamic monitoring in critically ill patients.
\end{abstract}

\section{Introduction}

Hemodynamic monitoring plays an important role in the management of today's acutely ill patient. Essentially, hemodynamic monitoring can be helpful in two key settings. The first is when a problem has been recognized; here, monitoring can help to identify underlying pathophysiological processes so that appropriate forms of therapy can be selected. A typical scenario is the patient in shock for whom options are to give more fluids or to give a vasopressor or an inotropic agent, depending on the hemodynamic evaluation. The second setting is more preventative, with monitoring allowing preemptive actions to be performed before a significant problem arises. A typical scenario here is the perioperative patient in whom monitoring can be used to detect hypovolemia

\footnotetext{
*Correspondence: Jvincen@ulb.ac.be

'Department of Intensive Care, Erasme Hospital, Université Libre de Bruxelles, 808 route de Lennik, 1070-Brussels, Belgium

Full list of author information is available at the end of the article
}

or low oxygen delivery $\left(\mathrm{DO}_{2}\right)$ early, enabling timely corrective therapy to be initiated.

Although microcirculatory changes are believed to play a major role in the development of organ dysfunction and multiple organ failure and there is increasing interest in new techniques to monitor the microcirculation, these are not yet available for clinical practice, and hemodynamic monitoring, therefore, still focuses on the macrocirculation. Current hemodynamic monitoring therefore includes measurement of heart rate, arterial pressure, cardiac filling pressures or volumes, cardiac output, and mixed venous oxygen saturation $\left(\mathrm{SvO}_{2}\right)$. Although not perfect, the pulmonary artery catheter (PAC) has long been considered the optimal form of hemodynamic monitoring, allowing for the almost continuous, simultaneous recording of pulmonary artery and cardiac filling pressures, cardiac output and $\mathrm{SvO}_{2}$. However, although the incidence of complications with the PAC is relatively low, the technique is still quite invasive and there is no clear evidence for improved outcomes associated with its insertion and use to guide therapy [1]. As a result, interest in alternative monitoring systems has surged in recent years.

There are now many different monitoring systems available, and physicians may feel somewhat confused by the multiple possibilities. These systems can be easily listed in order of degree of invasiveness, from the highly invasive PAC to the completely non-invasive bioimpedance/ bioreactance technique and transthoracic echo-Doppler. Classifying them according to how accurate (closeness of measured values to the 'true' value, expressed as the bias) or precise (variability of values due to random errors of measurement) [2] they are is more difficult, in part because of the lack of a perfect 'gold' standard for comparison. Most devices have been evaluated by comparing their results with those obtained by intermittent thermodilution from the PAC as the reference, although this technique has its own limitations and may not represent the best choice of comparator [2].

Our purpose in this consensus article is not to review the technology or modus operandi of the various systems 
Table 1. Examples of available methods to measure cardiac output

\begin{tabular}{|c|c|c|}
\hline Method & System & Limitations \\
\hline Thermodilution & PAC & Invasiveness - training required \\
\hline \multirow{8}{*}{$\begin{array}{l}\text { Transpulmonary } \\
\text { indicator dilution }\end{array}$} & $\mathrm{PiCCO}^{\oplus}$ & Decreased accuracy? \\
\hline & & Need for dedicated arterial catheter \\
\hline & $\mathrm{LiDCO}^{\mathrm{TM}}$ & Decreased accuracy? \\
\hline & & Need for lithium injection \\
\hline & & $\begin{array}{l}\text { Interference by non-depolarizing muscle relaxants; inaccurate in case of } \\
\text { hyponatremia }\end{array}$ \\
\hline & COstatus $^{\oplus}$ & Decreased accuracy? \\
\hline & VolumeView ${ }^{\mathrm{TM}}$ & Decreased accuracy? \\
\hline & & Need for dedicated arterial catheter \\
\hline $\begin{array}{l}\text { Arterial-pressure } \\
\text { waveform-derived }\end{array}$ & $\begin{array}{l}\mathrm{PiCCO}^{\oplus}, \mathrm{LiDCO}^{\mathrm{TM}}, \text { Vigileo }^{\mathrm{TM}} \\
\text { MostCare }\end{array}$ & Decreased accuracy, need for optimal arterial pressure tracing \\
\hline Esophageal Doppler & CardioQ $^{\text {TM }}$, WAKle TO & Training required, intermittent measurement \\
\hline Suprasternal Doppler & $\mathrm{USCOM}^{\oplus}$ & Difficult in some patients \\
\hline Echocardiography & $\begin{array}{l}\text { Vivid }^{\mathrm{TM}} \text {, Sonosite MicroMaxx } \\
\text { Philips } C \times 50^{\mathrm{TM}} \text {, and so on }\end{array}$ & Training required, intermittent measurement \\
\hline Partial $\mathrm{CO}_{2}$ rebreathing & $\mathrm{NiCO}^{\oplus}$ & Less reliable in respiratory failure \\
\hline Bioimpedance & $\begin{array}{l}\text { Lifegard }^{\oplus}, \text { TEBCO}^{\oplus}, \text { Hotman }^{\oplus} \text {, } \\
\text { BioZ }{ }^{\oplus} \text {, and so on }\end{array}$ & Less reliable in critically ill patients, not applicable in cardiothoracic surgery \\
\hline Bioreactance & $\mathrm{NICOM}^{\ominus}$ & Validated in only one study in critically ill patients \\
\hline
\end{tabular}

PAC, pulmonary artery catheter.

in any detail, not to provide readers with a shopping list, nor to identify one system that would be suitable in all patients; rather, we will briefly review the advantages and limitations of each system, and propose ten key principles to guide choice of monitoring system(s) in today's acutely ill patients.

\section{Available systems for monitoring cardiac output}

Examples of the main systems that are available for estimating cardiac output are listed in Table 1.

\section{Thermodilution (pulmonary artery catheter)}

The intermittent thermodilution technique, in which boluses of ice-cold fluid are injected into the right atrium via a PAC and the change in temperature detected in the blood of the pulmonary artery used to calculate cardiac output, is still widely considered as the standard method of reference. Adaptation of the PAC to incorporate a thermal filament (Vigilance ${ }^{\mathrm{mm}}$, Edwards Life Sciences, Irvine, CA, USA) or thermal coil $\left(\mathrm{OptiQ}^{\mathrm{TM}}\right.$, ICU Medical, San Clemente, CA, USA) that warms blood in the superior vena cava and measures changes in blood temperature at the PAC tip using a thermistor, provides a continuous measure of the trend in cardiac output, with the displayed values representing an average of the values over the previous 10 minutes. The averaged values have the advantage of eliminating variability in the presence of arrhythmias, but the disadvantage of not being real-time values, thus limiting the usefulness of this approach for assessing rapid hemodynamic changes in unstable patients.

The PAC has a key advantage over many other systems in that it provides simultaneous measurements of other hemodynamic parameters in addition to cardiac output, including pulmonary artery pressures, right-sided and left-sided filling pressures, and $\mathrm{SvO}_{2}$.

\section{Transpulmonary or ultrasound indicator dilution}

The $\mathrm{PiCCO}^{\circ}$ (Pulsion Medical Systems, Munich, Germany), LiDCO $^{\mathrm{mm}}$ (LiDCO Ltd, London, UK), VolumeView ${ }^{\mathrm{mm}}$ (Edwards Life Sciences), and COstatus ${ }^{\circledR}$ (Transonic Systems Inc., Ithaca, NY, USA) systems allow cardiac output to be investigated less invasively, using a central venous (to allow calibration) and an arterial catheter, rather than needing to introduce a catheter into the pulmonary artery. The $\mathrm{PiCCO}^{\circ}$ and recently launched VolumeView ${ }^{\mathrm{Tm}}$ systems require a femoral artery catheter. These devices use the same basic principles of dilution to estimate the cardiac output as with PAC thermodilution. $\mathrm{PiCCO}^{\circ}$ and VolumeView $^{\mathrm{Tm}}$ use injections of ice cold intravenous fluid 
as the indicator, measuring change in temperature downstream to calculate cardiac output, whereas $\mathrm{LiDCO}^{\mathrm{Tm}}$ uses minute amounts of lithium chloride as the indicator and measures levels using a lithium-selective electrode. COstatus $^{\bullet}$ calculates cardiac output by using ultrasound technology to measure changes in blood ultrasound velocity and blood flow following an injection of warm saline solution.

Cardiac output values measured using transpulmonary or ultrasound indicator dilution techniques correlate well with those measured using PAC thermodilution [3-6] and may show less respiratory phase-dependent variation [4]. The $\mathrm{PiCCO}^{\circ}$ and the VolumeView ${ }^{\mathrm{TM}}$ systems provide variables in addition to cardiac output, such as global end-diastolic volume and measurements of extravascular lung water. The COstatus ${ }^{\bullet}$ system also provides some derived variables, including total end diastolic volume index.

\section{Arterial pressure trace-derived estimation of cardiac output}

In addition to the intermittent indicator dilution cardiac output measurements discussed above, the $\mathrm{PiCCO}^{\circ}$ and $\mathrm{LiDCO}^{\mathrm{TM}}$ systems can also estimate cardiac output on a continuous basis from the arterial pressure waveform with $\left(\mathrm{PiCCO}^{\circ}\right.$ and $\mathrm{LiDCOplus}^{\mathrm{TM}}$ ) or without (LidCOrapid ${ }^{\mathrm{TM}}$ ) the need for recalibration when changes in vascular compliance may have occurred. The $\mathrm{PiCCO}^{\circ}$ system uses a pulse contour analysis and the $\mathrm{LiDCO}^{\mathrm{Tm}}$ system a pulse power analysis. In addition to these, Vigileo ${ }^{\mathrm{Tm}}$ (Edwards Life Sciences) and MostCare ${ }^{\mathrm{Tm}}$ (Vytech, Padova, Italy, using the Pressure Recording Analytical Method (PRAM)) systems have been developed for arterial waveform analysis without external calibration. Each of these systems contains a proprietary algorithm for converting a pressure-based signal into a flow measurement. The specific algorithms have individual characteristics and make different assumptions - for example, related to arterial compliance $\left(\right.$ Vigileo $^{\mathrm{TM}}$ ) or pressure $\left(\right.$ MostCare $^{\mathrm{TM}}$ ) which can make them more or less accurate depending on the clinical circumstances. The level of accuracy and precision of each device needs to be understood as the data cannot be superimposed from one system to another. The advantages of these arterial pressure-based cardiac output monitoring systems over PAC-derived measurements is primarily their less invasive nature.

The major weakness of all these devices is the drift in values whenever there is a major change in vascular compliance, as, for example, in vascular leak syndrome with increased vessel wall edema leading to decreased arterial compliance. Aortic valve regurgitation may further decrease the accuracy of these techniques. Overor under-damped arterial pressure waveforms will also decrease the precision of these monitors.

\section{Echocardiography and echo-Doppler}

Echocardiography allows measurement of cardiac output using standard two-dimensional imaging or, more commonly, Doppler-based methods. The main interest in echocardiography in general is that it can be used not only for measurement of cardiac output but also for the additional assessment of cardiac function. Echocardiography is particularly useful as a diagnostic tool because it allows the visualization of cardiac chambers, valves and pericardium. Small ventricles ('kissing ventricles') may incite fluid administration whereas a poorly contractile myocardium may suggest that a dobutamine infusion is a better choice. Right ventricular dilatation may orient towards the diagnosis of massive pulmonary embolism or myocardial infarction whereas the presence of pericardial fluid may suggest a diagnosis of pericardial tamponade. Severe valvulopathy can also be recognized promptly. However, echocardiography instruments and expertise may not be readily available everywhere; in some institutions, this is still the domain of the cardiologists and they need to be called to do the procedure.

If an ultrasound beam is directed along the aorta using a probe, part of the ultrasound signal will be reflected back by the moving red blood cells at a different frequency. The resultant Doppler shift in the frequency can be used to calculate the flow velocity and volume and hence cardiac output. Echo-Doppler evaluation can provide reasonable estimates of cardiac output, but again is operatordependent and continuous measurement of cardiac output using this technique is not possible. Echo-Doppler evaluation may be applied either transthoracically or transesophageally. However, transthoracic techniques do not always yield good images and transesophageal techniques are more invasive such that some sedation, and often endotracheal intubation, is required in order to obtain reliable measurements. Moreover, the esophageal probe is uncomfortable in non-intubated patients, although may be better tolerated if inserted nasally, and should be used cautiously in patients with esophageal lesions. The signal produces different waveforms that can be used to distinguish to some extent changes in preload, afterload and left ventricular contractility. Doppler flow studies focusing on the descending thoracic aorta may not provide a reliable measurement of the total cardiac output (for example, with epidural use), and are invalid in the presence of intra-aortic balloon pumping. Echo-Doppler cardiac output estimates vary considerably for several reasons, including difficulty in assessment of the velocity time integral, calculation error due to the angle of insonation, and problems with correct measurement of the cross-sectional area. Some training is required when using these techniques. Esophageal-Doppler techniques have been shown to be useful for optimizing fluid administration in high risk surgical patients $[7,8]$. 
Simplified transesophageal Doppler techniques can be convenient as the probe is smaller than for standard esophageal echocardiography techniques. Simplified transthoracic Doppler systems allow estimation of aortic blood flow and may be even less invasive; however, although these techniques can be simple to perform in healthy volunteers, access to good images may be more difficult in critically ill patients. Moreover, there is a fairly prolonged learning curve for correct use of this system [9]. These methods need further validation in critically ill patients.

\section{$\mathrm{CO}_{2}$ rebreathing}

$\mathrm{CO}_{2}$ rebreathing systems, based on the Fick principle, use a $\mathrm{CO}_{2}$ sensor, a disposable airflow sensor and a disposable rebreathing loop. $\mathrm{CO}_{2}$ production is calculated from minute ventilation and its $\mathrm{CO}_{2}$ content, and the arterial $\mathrm{CO}_{2}$ content is estimated from end-tidal $\mathrm{CO}_{2}$. Partial rebreathing reduces $\mathrm{CO}_{2}$ elimination and increases the end-tidal $\mathrm{CO}_{2}$. By combining measurements taken during and without rebreathing, venous $\mathrm{CO}_{2}$ content can be eliminated from the Fick equation. However, intrapulmonary shunting of blood and rapid hemodynamic changes affect the accuracy of the measurement, so that this technique is not considered to be reliable in acutely ill patients.

\section{Bioimpedance and bioreactance}

Bioimpedance is based on the fact that the conductivity of a high-frequency, low-magnitude alternating current passed across the thorax changes as blood flow varies with each cardiac cycle. These changes can be measured using electrodes placed on a patient's chest and used to generate a waveform from which cardiac output can be calculated. Bioreactance has developed out of bioimpedance and measures changes in the frequency of the electrical currents traversing the chest, rather than changes in impedance, potentially making it less sensitive to noise. These techniques are non-invasive and can be applied quickly. They have been used for physiological studies in healthy individuals and may be useful in perioperative applications [10], but are less reliable in critically ill patients [11]. Electrical interference may also occur in the ICU environment.

\section{Key principles of hemodynamic monitoring}

Having briefly discussed some of the advantages and limitations of the available systems, we now consider some key principles than can help when considering which hemodynamic monitoring system to use.

\section{Principle 1: no hemodynamic monitoring technique can improve outcome by itself}

Hemodynamic monitoring can only improve outcomes if three conditions are met: the data obtained from the monitoring device must be sufficiently accurate to be able to influence therapeutic decision making; the data obtained from the monitoring system must be relevant to the patient being monitored; and changes in management made as a result of the data obtained need to be able to improve outcomes. If the data are interpreted or applied incorrectly, or the therapies themselves are ineffective or harmful, the resultant change in management will not improve patient status and may be deleterious.

If these three conditions are not met, monitoring is unlikely to be associated with improved outcomes, and this may account for the lack of evidence of improved outcomes in acutely ill patients with use of any monitoring device, not only the PAC [12].

\section{Principle 2: monitoring requirements may vary over time and can depend on local equipment availability and training}

The optimal monitoring system will depend on the individual patient, the problem already present or potentially arising for which the monitoring is required, and the devices and expertise available at the institution in question.

For initial evaluation of the critically ill patient, an invasive approach is still often needed, which includes insertion of an arterial catheter and a central venous catheter; this is because of the need for secure intravenous and arterial access in such patients and the presumed increased accuracy of measurements based on direct pressure monitoring. The data provided can already guide initial treatment. Analysis of the arterial pressure trace can identify fluid responsiveness in mechanically ventilated patients, although there are some limitations to this technique, including adaptation to the respirator (often with high doses of sedative agents and even paralysis), need for absence of arrhythmias, and use of relatively large tidal volumes. Response to passive leg raising can be used if beat-by-beat measurements of stroke volume are monitored. Once stabilized, less invasive monitoring techniques should be employed. Importantly, monitoring systems are not necessarily mutually exclusive and can sometimes be used to complement each other. For example, echocardiography can provide additional information in the early assessment of critically ill patients (Figure 1).

There is still a place for the PAC (Swan-Ganz), which has the advantage of allowing measurement of cardiac filling pressures and pulmonary artery pressures, cardiac output and $\mathrm{SvO}_{2}$ (and now also extravascular lung water). However, although in the past a PAC was inserted early in all critically ill patients, today its insertion is no longer necessary during initial resuscitation, but should rather be reserved for complex cases, for example, patients with right ventricular dysfunction, difficult assessment of 


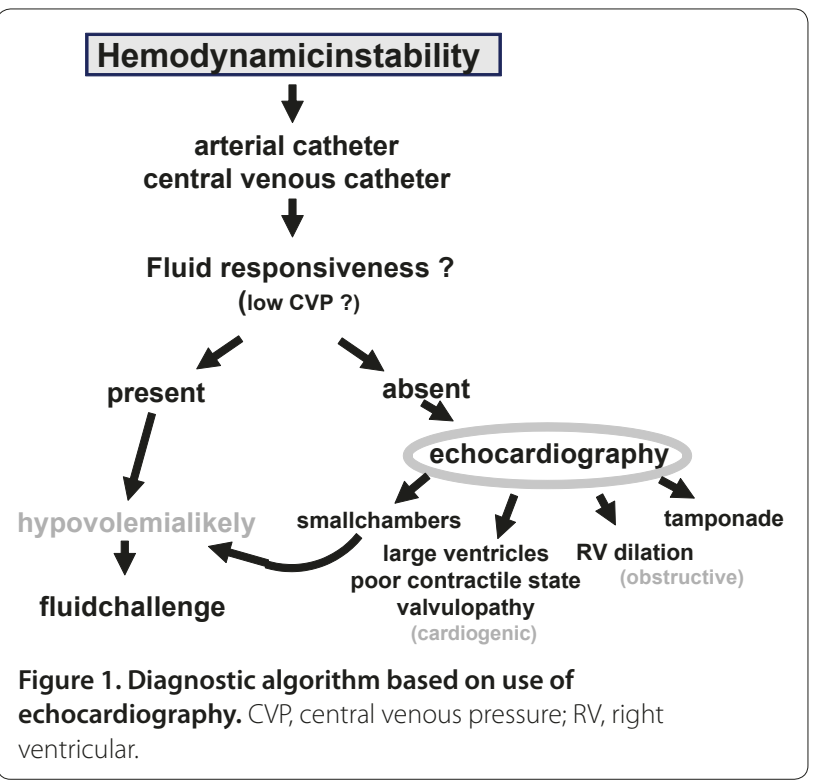

optimal fluid management, or specific cases of cardiac failure.

Principle 3: there are no optimal hemodynamic values that are applicable to all patients

Although it may be appealing to have some simple targets, such as keeping the mean arterial pressure above $65 \mathrm{mmHg}$, the central venous pressure (CVP) above $8 \mathrm{mmHg}$, or $\mathrm{DO}_{2}$ above $600 \mathrm{~mL} /$ minute/ $\mathrm{M}^{2}$, such targets are overly simplistic and may even be potentially dangerous. For example, the acceptable minimal arterial pressure may be very different in a young individual without co-morbidity compared to an elderly atherosclerotic, previously hypertensive patient. Likewise, the CVP may remain low in adequately resuscitated patients or may be high at baseline in patients with pulmonary hypertension due to underlying chronic lung disease. Similarly, it is difficult to define an optimal level of cardiac output as cardiac output is an adaptative parameter for which there is no single 'normal' value, but only normal ranges. Since the purpose of the cardiovascular system is to match blood flow to metabolic demand, and this demand may vary widely even over short time intervals, targeting a specific cardiac output or even sustaining a threshold value may be inappropriate. For example, keeping a cardiac output above 5 or 6 liters per minute in an adult would be like driving constantly at $80 \mathrm{~km} / \mathrm{h}$, whether in a small town or on the freeway. In the critically ill, cardiac output increases in sepsis, as in anemia, but may be reduced with sedation or anesthesia. Multiple factors therefore need to be considered when determining whether cardiac output is optimal for a particular patient, including the degree of tissue perfusion as estimated from a careful clinical examination

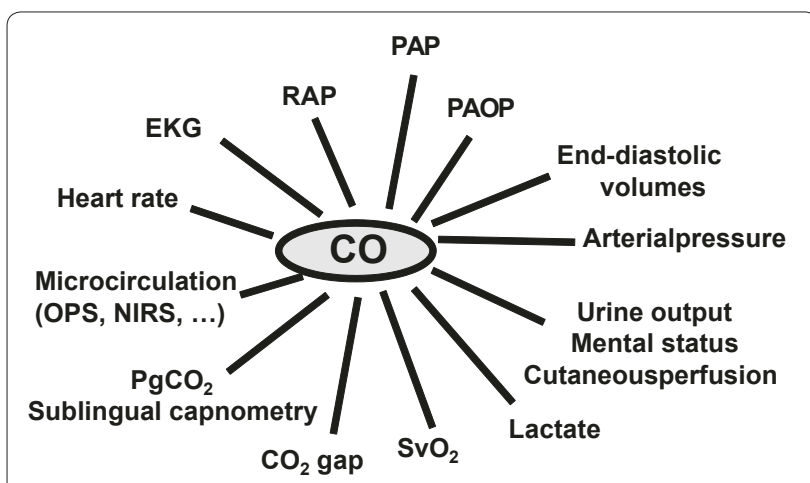

Figure 2. Factors influencing the interpretation of cardiac output (CO). EKG, electrocardiogram; NIRS, near-infrared spectral imaging; OPS, orthogonal polarization spectral imaging; PAOP, pulmonary artery occlusion pressure; PAP, pulmonary artery pressure; $\mathrm{PgCO}_{2^{\prime}}$ gastric intramucosal carbon dioxide partial pressure; RAP, right atrial pressure; $\mathrm{SvO}_{2}$, mixed venous oxygen saturation.

and blood lactate levels (Figure 2). Alarms should thus be individualized for each patient and reevaluated regularly.

Principle 4: we need to combine and integrate variables

Any variable on its own provides relatively little information - it is just one piece of a large puzzle. We need rather to integrate all the available data from multiple sources. For example, a hypotensive patient with a low cardiac output will present different diagnoses (hypovolemia, decreased contractility or obstruction) and hence require different treatments to a hypotensive patient with a high cardiac output (decreased vascular tone). Likewise, as discussed earlier, correct interpretation of a low cardiac output involves consideration of many factors (Figure 2), including some assessment of cardiac filling (pressures or dimensions) to assess ventricular preload.

\section{Principle 5: measurements of $\mathrm{SvO}_{2}$ can be helpful}

$\mathrm{SvO}_{2}$ reflects the balance between oxygen consumption $\left(\mathrm{VO}_{2}\right)$ and $\mathrm{DO}_{2}$ and thus provides an indication of the adequacy of tissue oxygenation. If there is no PAC in situ, the oxygen saturation in the superior vena cava $\left(\mathrm{ScvO}_{2}\right)$ can be measured using a central venous catheter and has been proposed as a surrogate for $\mathrm{SvO}_{2}$. Importantly, $\mathrm{ScvO}_{2}$ represents just an approximation of the $\mathrm{SvO}_{2}$ [13] and the absolute values of $\mathrm{ScvO}_{2}$ and $\mathrm{SvO}_{2}$ are not interchangeable. The difference between these two parameters is influenced by the sampling site of central venous blood, the presence of left-to-right shunts, incomplete mixing of venous blood, oxygen extraction in the renal and the splanchnic beds, redistribution of blood flow through the upper and lower body, level of consciousness (anesthesia) and myocardial $\mathrm{VO}_{2}$. The reliability of $\mathrm{ScvO}_{2}$ is also dependent on the position of the tip of the catheter, with right atrial measurements closely 


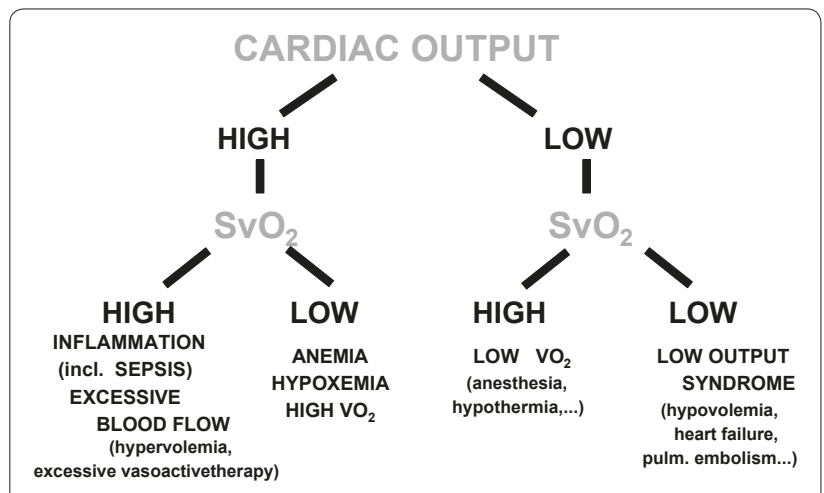

Figure 3. Diagnostic algorithm based on mixed venous oxygen saturation $\left(\mathrm{SvO}_{2}\right)$ and cardiac output. $\mathrm{VO}_{2^{\prime}}$ oxygen consumption.

approximating $\mathrm{SvO}_{2}$ and high vena cava measurements often deviating substantially from $\mathrm{SvO}_{2}$. In general, $\mathrm{SvO}_{2}$ is more useful when the value is below normal (see below), even though in these conditions it may not reflect a hemodynamic problem. Simultaneous measurements of blood lactate levels can be helpful. A diagnostic algorithm based on $\mathrm{SvO}_{2}$ and cardiac output is shown in Figure 3.

\section{Principle 6: a high cardiac output and a high $\mathrm{SvO}_{2}$ are not always best}

Although ICU physicians may like to increase cardiac output and $\mathrm{SvO}_{2}$ by giving more fluid and inotropic agents, is this always good? Excessive fluid administration to increase cardiac output may result in fluid overload with massive edema formation and this may be associated with worse outcomes [14]; some systems measure extravascular lung water, which can help document this. Similarly, excessive doses of dobutamine can be detrimental, compromising myocardial function, especially in patients with coronary artery disease; giving inotropic agents in the presence of coronary artery disease is like trying to stimulate a tired horse. Using vasoactive agents and fluids to increase $\mathrm{DO}_{2}$ to supranormal levels in all patients may result in excessive mortality rates and this strategy has been abandoned [15]. A high $\mathrm{ScvO}_{2}$ has been suggested as a target for some high risk patients or in shock resuscitation, with Rivers and colleagues [16] reporting that septic patients assigned to an early goaldirected therapy algorithm had higher $\mathrm{ScvO}_{2}$ values and reduced mortality rates. However, this was a strategy for early resuscitation of patients with severe sepsis in a single institution, and needs further validation in multicenter studies, several of which are currently ongoing. Importantly, applying the same strategy in general ICU patients may not improve outcomes [17]. Indeed, in patients with sepsis, a high $\mathrm{SvO}_{2}$ may be the result of maldistribution of peripheral blood flow and altered oxygen extraction, rather than adequate perfusion, such that patients may still deteriorate even with a high $\mathrm{SvO}_{2}$. In sepsis, a high cardiac output, like a high $\mathrm{SvO}_{2}$, can be associated with worse outcomes.

\section{Principle 7: cardiac output is estimated, not measured}

No bedside method is available to directly assess cardiac output, so all values obtained are estimates. As such, comparison of measurements obtained with different techniques results in relatively poor agreement and significant bias. The intermittent thermodilution technique is generally considered as the 'reference' standard, but has its own limitations. A measurement obtained by a less invasive technique may be preferable if it can be obtained more rapidly and easily, even if it is slightly less accurate. Importantly, the accuracy of absolute values may be less important if one is following trends, for example, to track the short-term effects of therapies, such as fluid loading.

\section{Principle 8: monitoring hemodynamic changes over short periods of time is important}

Monitoring of acute changes in cardiac output can be important, for example, in patients at risk of acute bleeding or in assessing the response to fluid administration to separate fluid responders from non-responders. Evaluating the response to a dobutamine or to a nitrate infusion is another example of this functional monitoring that may also have sound clinical applications. This assessment of hemodynamic variations observed during the challenge of the cardiovascular system has been termed 'functional hemodynamic monitoring' [18]. The study of slow changes in cardiac output over several days may be less relevant in most patients, although can be useful to follow the clinical course of the cardiac patient. Combining measures of multiple variables and their dynamic interactions in response to time and specific treatments often increases the sensitivity and specificity of these monitoring modalities to identify specific disease processes and quantify whether therapy is effective or not.

\section{Principle 9: continuous measurement of all hemodynamic variables is preferable}

Although there are no data to demonstrate the superiority of continuous cardiac output measurements over intermittent monitoring, there has been a global evolution towards more continuous measurement of variables. We can now routinely measure various hemodynamic variables continuously, including heart rate, arterial pressure and CVP. Using the thermodilution technique, one may not wish to go back to intermittent measurements of cardiac output by repeated injections of cold water boluses. It may even be preferable to have realtime (beat-by-beat) continuous cardiac output measurement rather than a built-in delay like the semi-continuous 
Table 2. The key properties of an 'ideal' hemodynamic monitoring system

Provides measurement of relevant variables
Provides accurate and reproducible measurements
Provides interpretable data
Is easy to use
Is readily available
Is operator-independent
Has a rapid response-time
Causes no harm
Is cost-effective
Should provide information that is able to guide therapy

cardiac output provided with the Vigilance ${ }^{\mathrm{min}}$ system. Systems that are not continuous (for example, echocardiography) or that require calibration (for example, transpulmonary indicator dilution) may not provide the real-time data needed for optimal acute management of unstable critically ill patients, whereas systems that provide continuous non-calibrated cardiac output measurements suffer from reduced accuracy.

\section{Principle 10: non-invasiveness is not the only issue}

Non-invasiveness is not the only goal. Although it is always preferable to be less invasive, being non-invasive is not always possible and may even be counter effective. For example, continuous monitoring of arterial pressure is more invasive than intermittent monitoring but is helpful in hypotensive (or severe hypertensive) states. Likewise, a central venous catheter can be helpful to monitor the CVP and the $\mathrm{ScvO}_{2}$ (and also facilitates the rapid administration of fluids). Whenever possible, we should of course try to be as non-invasive as possible, but arterial pressure monitoring and CVP monitoring are still invasive. Echocardiography must be promoted more for its ability to offer a direct evaluation of cardiac function than for its non-invasiveness. Even though it is the most invasive method, the PAC is still of value in very sick patients with complex problems, for example, respiratory failure with shock and oliguria. At the other extreme, completely non-invasive bioimpedance has a place in healthy individuals, but little place in critically ill patients. Other monitoring systems are of use in patients with conditions somewhere between these two extremes. The optimal device depends on the type of patient, the question being asked, and the condition being managed or anticipated.

\section{Conclusion}

The ideal hemodynamic monitoring system should comprise all the key factors listed in Table 2; however, such a system does not currently exist so we must try and choose devices that have a maximum of these attributes, bearing in mind that there is no 'one size fits all' type of system and one should, therefore, select the system most appropriate for each patient and, perhaps even more importantly, for each type of problem. It is important to be familiar with the technology being used, profiting from its advantages but recognizing its limitations. Most systems now offer (almost) continuous measurements, with the possible exception of echocardiography techniques because it is difficult to leave the probe in place for prolonged periods. Hemodynamic monitoring can be particularly helpful in the early stages of resuscitation, but is less useful when organ failure is established. Most importantly, one must never forget that it is not the monitoring itself that can improve outcomes but the changes in therapy guided by the data obtained.

\section{Abbreviations}

$\mathrm{CVP}$, central venous pressure; $\mathrm{DO}_{2^{\prime}}$ oxygen delivery; $\mathrm{PAC}$, pulmonary artery catheter; $\mathrm{ScvO}_{2}$, central venous oxygen saturation; $\mathrm{SvO}_{2}$, mixed venous oxygen saturation; $\mathrm{VO}_{2^{\prime}}$ oxygen consumption.

\section{Competing interests}

J-LV has received grants from Edwards Lifesciences and Pulsion Medical Systems. AR has received lecture fees from LiDCO and advisory board fees from Cheetah and Edwards. AP is a member of the medical advisory board of Pulsion Medical Systems and consultant to BMEYE. GSM is a member of the medical advisory board of Pulsion Medical Systems. GDR has no conflicts related to this manuscript. BV is an advisor to, and has received lecture fees from, Edwards. MRP is a paid advisor to LiDCO Ltd, Edwards LifeSciences Inc., and Applied Physiology; he has stock options with LiDCO and Cheetah Medical and has received honoraria from Cheetah Medical. CKH has received lecture fees and research grants from Pulsion Medical Systems and Edwards Lifesciences. J-LT is a member of the medical advisory board of Pulsion Medical Systems. W-PdeB has received research grants from Transonic Systems Inc. and Pulsion Medical Systems. SS has received research grants from Vygon and Vythec. AV-B has received research grants from General Electric and Maquet. DDeB has received grants and material for studies from Edwards Lifesciences, Pulsion Medical Systems, LiDCO and Vytech and honoraria for lectures from Edwards Lifesciences and Pulsion Medical Systems. KRW has no conflicts related to this manuscript. MM is a member of the medical advisory board of Pulsion Medical Systems. MS is on the Advisory Board for Deltex Medical and Covidien; Deltex Medical provide unrestricted research grants.

\section{Author details}

'Department of Intensive Care, Erasme Hospital, Université Libre de Bruxelles, 808 route de Lennik, 1070-Brussels, Belgium. ${ }^{2}$ Department of Intensive Care Medicine, St George's Healthcare NHS Trust, Blackshaw Road, London, SW17 0QT, UK. ' ${ }^{3}$ epartment of Anesthesiology and Intensive Care, Sheba Medical Center, Tel Aviv University, Tel Aviv, 52621 Israel. "ADivision of Pulmonary, Allergy and Critical Care, Emory University School of Medicine, Grady Memorial Hospital, 615 Michael Street, Suite 205, Atlanta, GA 30322, USA. ${ }^{5}$ Department of Anesthesia and Intensive Care Medicine, University Hospital, Medical School, University of Udine, P.le S. Maria della Misericordia, 1533100 Udine, Italy. ${ }^{6}$ Department of Anesthesiology and Critical Care Medicine, Pôle d'Anesthesie Reanimation, Hôpital Claude Huriez, rue Michel Polonoski, CHU Univ Nord de France, 59000 Lille, France. 'Department of Critical Care Medicine, 606 Scaife Hall, 3550 Terrace Street, Pittsburgh, PA 15261, USA. Institute of Anaesthesiology and Intensive Care Medicine, Triemli City Hospital Zurich, Birmensdorferstr. 497, 8063 Zurich, Switzerland. ${ }^{9}$ Service de réanimation médicale, Centre Hospitalo-Universitaire de Bicêtre, Assistance Publique-Hôpitaux de Paris, EA 4046, Université Paris Sud, 78 rue du Général Leclerc, 94270 Le Kremlin-Bicêtre, France. ${ }^{10}$ Department of Neonatology, Radboud University Nijmegen Medical center, PO Box 9101, 6500 Nijmegen, The Netherlands. "'Department of Anaesthesia and Intensive Care, University of Siena, Viale Bracci, 1, 53100 Siena, Italy. ${ }^{12}$ Réanimation 
médicale, CHU Ambroise Paré, 9 avenue Charles-de-Gaulle, 92104 Boulogne, France. ${ }^{13} \mathrm{Critical}$ Care Research Laboratories, Heart and Lung Institute, University of British Columbia, 166-1081 Burrard Street, Vancouver, British Columbia, V6Z 1Y6, Canada. ${ }^{14}$ Intensive Care Unit, Department of Internal Medicine, University Hospital Zurich, Raemistrasse 100, CH-8091 Zurich, Switzerland. ${ }^{15}$ Department of Intensive Care, University College London, Cruciform Building, Gower Street, London, WC1E 6BT, UK.

Published: 18 August 2011

\section{References}

1. Shah MR, Hasselblad V, Stevenson LW, Binanay C, O'Connor CM, Sopko G, Califf RM: Impact of the pulmonary artery catheter in critically ill patients: meta-analysis of randomized clinical trials. JAMA 2005, 294:1664-1670.

2. Squara P, Cecconi M, Rhodes A, Singer M, Chiche JD: Tracking changes in cardiac output: methodological considerations for the validation of monitoring devices. Intensive Care Med 2009, 35:1801-1808.

3. Goedje O, Hoeke K, Lichtwarck-Aschoff M, Faltchauser A, Lamm P, Reichart B Continuous cardiac output by femoral arterial thermodilution calibrated pulse contour analysis: comparison with pulmonary arterial thermodilution. Crit Care Med 1999, 27:2407-2412.

4. Sakka SG, Reinhart K, Meier-Hellmann A: Comparison of pulmonary artery and arterial thermodilution cardiac output in critically ill patients. Intensive Care Med 1999, 25:843-846.

5. Costa MG, Della Rocca G, Chiarandini P, Mattelig S, Pompei L, Barriga MS, Reynolds T, Cecconi M, Pietropaoli P: Continuous and intermittent cardiac output measurement in hyperdynamic conditions: pulmonary artery catheter vs. lithium dilution technique. Intensive Care Med 2008, 34:257-263.

6. Tsutsui M, Matsuoka N, Ikeda T, Sanjo Y, Kazama T: Comparison of a new cardiac output ultrasound dilution method with thermodilution technique in adult patients under general anesthesia. J Cardiothorac Vasc Anesth 2009, 23:835-840.

7. Wakeling HG, McFall MR, Jenkins CS, Woods WG, Miles WF, Barclay GR, Fleming SC: Intraoperative oesophageal Doppler guided fluid management shortens postoperative hospital stay after major bowel surgery. Br J Anaesth 2005, 95:634-642.

8. Abbas SM, Hill AG: Systematic review of the literature for the use of oesophageal Doppler monitor for fluid replacement in major abdominal surgery. Anaesthesia 2008, 63:44-51.
9. Lelyveld-Haas LE, van Zanten AR, Borm GF, Tjan DH: Clinical validation of the non-invasive cardiac output monitor USCOM-1A in critically ill patients. Eur J Anaesthesiol 2008, 25:917-924.

10. Marque S, Cariou A, Chiche JD, Squara P: Comparison between FlotracVigileo and Bioreactance, a totally noninvasive method for cardiac output monitoring. Crit Care 2009, 13:R73.

11. Squara P, Denjean D, Estagnasie P, Brusset A, Dib JC, Dubois C: Noninvasive cardiac output monitoring (NICOM): a clinical validation. Intensive Care Med 2007, 33:1191-1194.

12. Ospina-Tascon GA, Cordioli RL, Vincent JL: What type of monitoring has been shown to improve outcomes in acutely ill patients? Intensive Care Med 2008, 34:800-820.

13. Reinhart K, Kuhn HJ, Hartog C, Bredle DL: Continuous central venous and pulmonary artery oxygen saturation monitoring in the critically ill. Intensive Care Med 2004, 30:1572-1578.

14. Sakr Y, Vincent JL, Reinhart K, Groeneveld J, Michalopoulos A, Sprung CL, Artigas A, Ranieri VM: High tidal volume and positive fluid balance are associated with worse outcome in acute lung injury. Chest 2005, 128:3098-3108.

15. Dellinger RP, Levy MM, Carlet JM, Bion J, Parker MM, Jaeschke R, Reinhart K, Angus DC, Brun-Buisson C, Beale R, Calandra T, Dhainaut JF, Gerlach H, Harvey M, Marini JJ, Marshall J, Ranieri M, Ramsay G, Sevransky J, Thompson BT, Townsend S, Vender JS, Zimmerman JL, Vincent JL: Surviving Sepsis Campaign: international guidelines for management of severe sepsis and septic shock: 2008. Crit Care Med 2008, 36:296-327.

16. Rivers E, Nguyen B, Havstad S, Ressler J, Muzzin A, Knoblich B, Peterson E, Tomlanovich M: Early goal-directed therapy in the treatment of severe sepsis and septic shock. N Engl J Med 2001, 345:1368-1377.

17. Gattinoni L, Brazzi L, Pelosi P, Latini R, Tognoni G, Pesenti A, Fumagalli R: A trial of goal-oriented hemodynamic therapy in critically ill patients. SvO2 Collaborative Group. N Engl J Med 1995, 333:1025-1032.

18. Pinsky MR, Payen D: Functional hemodynamic monitoring. Crit Care 2005, 9:566-572

doi:10.1186/cc10291

Cite this article as: Vincent $J L$, et al: Clinical review: Update on hemodynamic monitoring - a consensus of 16. Critical Care 2011, 15:229. 